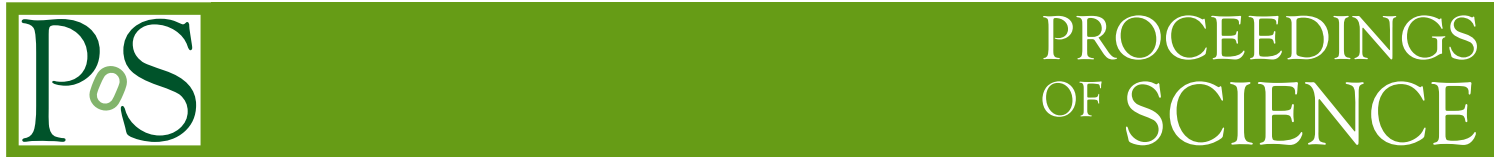

\title{
Hot QCD vs cosmology
}

\section{Laine*}

Institute for Theoretical Physics, Albert Einstein Center, University of Bern Sidlerstrasse 5, CH-3012 Bern, Switzerland

E-mail: laineditp.unibe.ch

It is a long-standing dream to "simulate" cosmology in laboratory through heavy ion collision experiments. Although the QCD epoch itself may not leave major cosmological signatures, theoretical methods developed and tested in the context of heavy ion collision experiments could indeed find applications at other energy scales. Here recent progress in this spirit is reviewed.

Xth Quark Confinement and the Hadron Spectrum,

October 8-12, 2012

TUM Campus Garching, Munich, Germany

\footnotetext{
${ }^{*}$ Speaker.
} 


\section{Introduction}

Among the main motivations for carrying out heavy ion collision experiments, at least according to statements made to the general public, is the aim to recreate for a brief moment conditions similar to those that existed in the early universe a few microseconds after the Big Bang. The corresponding temperature range is just below one $\mathrm{GeV}$, and it was originally thought that in this temperature range QCD might undergo a strong first order phase transition, separating the hadronic world from a partonic one. If so, the cosmological QCD transition could conceivably have left behind remarkable signatures (see e.g. ref. [1]), and reproducing any of the corresponding dynamics in the laboratory would have been a major achievement.

Unfortunately, a more precise understanding of thermal QCD, obtained largely through numerical lattice Monte Carlo simulations in the last two decades, has led to the conclusion that QCD possesses no strong first order phase transition. In fact, most likely it possesses no phase transition of any kind, only a smooth crossover [2,3]. (Even in the unlikely case that some transition were to be found after further refinements, it will be too weak for cosmological significance.)

The absence of a phase transition is a "problem" because it makes it unlikely that the QCD epoch could have cosmological significance. The reason is that thermodynamic equilibrium is a state which has no memory; all properties of the system are determined by the then temperature. Therefore cosmological relics rely on deviations from equilibrium (cartoons of typical scenarios for deviating from equilibrium are shown in fig. 1). But, in the absence of a phase transition, all QCD degrees of freedom interact fast enough to remain in equilibrium.

To be more concrete on this important point, let us recall that heavy ion collision experiments have indicated that QCD thermalizes within the time scale of a few fm/c. In cosmology around $T \sim 1 \mathrm{GeV}$, the time available is $m_{\mathrm{Pl}} / T^{2} \sim 10^{17} \mathrm{fm} / \mathrm{c}$, i.e. plenty enough to establish full equilibrium. So, whatever perturbations may be generated by thermal fluctuations are subsequently lost in time.

These remarks call for a modification of how we view the link between heavy ion collision experiments and cosmology. Indeed today the possibility of establishing a direct physics link between the two fields is, if not completely excluded, then at least restricted to specific cases (cf. sec. 2). In contrast, on a more abstract but also more general level, there is a very useful exchange of ideas (cf. sec. 3) and theoretical methods (cf. sec. 4) constantly taking place. In fact, as will be reviewed below, it is not too difficult to find examples of recent developments where techniques were first developed on the hot QCD side and subsequently exported to cosmology; and vice versa.
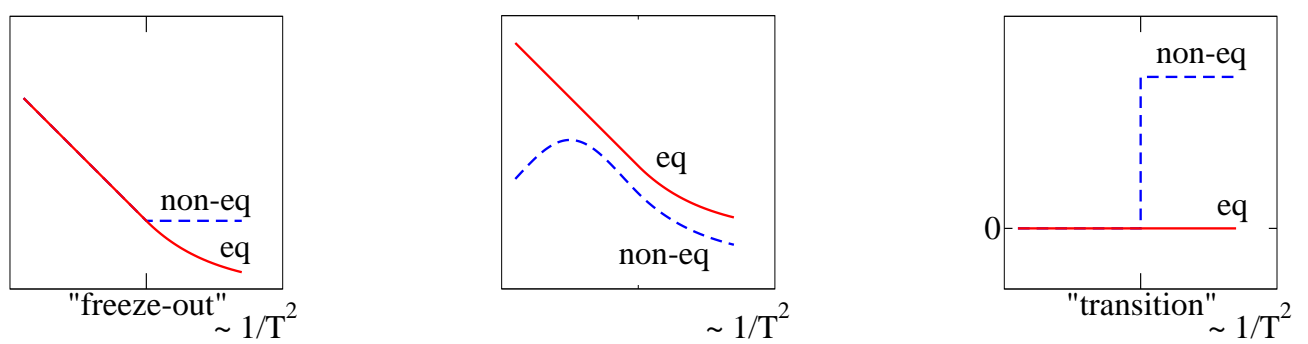

Figure 1: Various scenarios for falling out of thermal equilibrium in cosmology. Left: "freeze out" for dark matter, and "strong washout" regime for leptogenesis. Middle: origin of "non-thermal" dark matter, and "weak washout" regime for leptogenesis. Right: "phase transition" for baryogenesis. 


\section{A direct role for hot QCD in cosmology?}

Despite the remarks of sec. 1 , let us start by briefly recalling that specific possibilities do exist even for a direct link between hot QCD physics and cosmology. In these cases QCD-related particles do not themselves decouple from equilibrium, but their collective dynamics plays a background role for the evolution of other perturbations which just happen to be around.

As a first example in this direction, let us mention the properties of the primordial gravitational wave background. It is assumed that inflation generates a background with a certain "flat" spectrum. The spectrum observed today depends, however, also on the history of the Universe after the inflationary period; in particular it depends on how long a certain wavelength spends inside the horizon after re-entering it. This in turn is dictated by the thermal history and the equation of state of the matter filling the Universe. Consequently, the QCD epoch, during which the overall energy density and therefore the expansion rate changes by a significant amount, does lead to a distinctive feature in the gravitational wave spectrum that could in principle be observed $[4,5,6]$.

Another example is the problem of Dark Matter. Again, the main "signals" are the measurement of the Dark Matter relic density and, ultimately, the discovery of Dark Matter particles. However, in many scenarios Dark Matter gets generated through a freeze-out process as illustrated in fig. 1(left). The freeze-out process involves the competition of two rates, the expansion rate of the Universe and a microscopic "chemical equilibration rate" (cf. sec. 4.2) which tries to keep up with it. So, if the freeze-out happens around the QCD scale, the expansion rate leaves an imprint on the final number density [7]. The effect is probably most significant in the so-called $v \mathrm{MSM}$ scenario in which Dark Matter is of "non-thermal" type, cf. fig. 1(middle); the parameter space for this model is rather restricted by now (it is sketched in fig. 3 as the left-most island), but in principle still open $[8,9]$. Hot QCD related uncertainties in the corresponding computations, originating both from the equation of state as well as from the knowledge of hadronic spectral functions in various channels, were analyzed in ref. [10], and could in principle be reduced by further knowledge accumulated through heavy ion collision experiments and the associated lattice Monte Carlo simulations (for spectral functions see, e.g., ref. [11]).

\section{Exchange of ideas and concepts}

Moving then to a more abstract but also more general level, many of the basic ideas and concepts used in heavy ion collision experiments of course originate from cosmology. These include concepts such as the "Little Bang"; the paradigm of starting with a non-equilibrium state which then rapidly thermalizes; as well as the idea of modelling the thermal evolution through hydrodynamic expansion. Relatively recently, the idea has also emerged of systematically representing

heavy ion data in spherical harmonics in the same way as is routinely done in the analysis of the cosmic microwave background radiation. Without going into any details on this topic, on which the speaker is no expert, a random example of a recent suggestion for applying cosmological tools in heavy ion collision data analysis is shown in fig. 2.

\section{Exchange of theoretical methods}

The main focus of this talk is on the exchange of theoretical methods between the fields of hot 


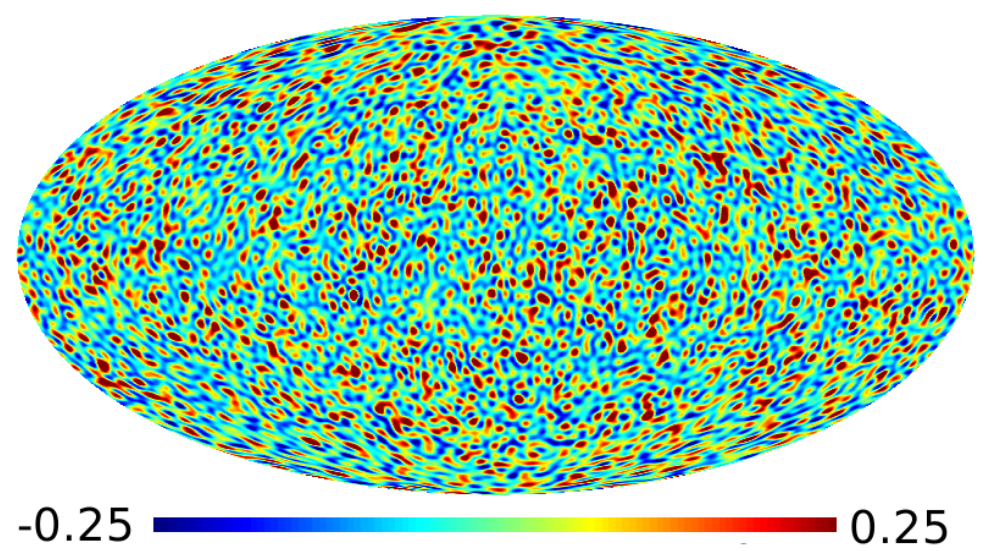

Figure 2: From ref. [12] (cf. also references therein). Shown is a HIJING simulation of an event of multiplicity 17000 , represented in a similar form as cosmic microwave background anisotropies in cosmology.

QCD and cosmology. From the conceptual point of view, the important observables of both cases can be classified in a similar way. The simplest class includes purely "static" quantities, namely thermal expectation values of components of the energy-momentum tensor, conserved currents, or gauge-invariant condensates. We refer to this class as the equation of state. The second class contains thermal expectation values of real-time 2-point correlation functions of the same opera-

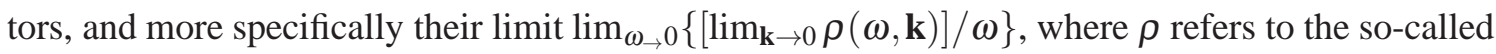
spectral function. This class is known as transport coefficients. In the third case we consider the spectral function at $\mathbf{k} \neq \mathbf{0}, \omega \neq 0$; then we can speak of thermal particle production. (If one particle species is being produced, rather than a resonance decaying into several particles, then $\omega$ is on-shell, i.e. $\omega=\sqrt{k^{2}+M^{2}}$, where $M$ denotes a physical mass.)

\subsection{Equation of state}

In cosmology, it is in general an excellent approximation to set the baryon chemical potential to zero, so that thermodynamic potentials are functions of the temperature, $T$, only. Then the comparison of the overall expansion rate and a chemical equilibration rate depends on $p(T), p^{\prime}(T)$ and $p^{\prime \prime}(T)$, where $p(T)$ denotes the pressure [13]. The same functions (save without the contribution of photons and leptons, which have no time to thermalize) dictate the expansion of the thermal fireball created in an energetic heavy ion collision. Hence the longstanding efforts, both analytic (via chiral perturbation theory at low temperatures, $T \ll 200 \mathrm{MeV}$, and the weak-coupling expansion at high temperatures, $T \gg 200 \mathrm{MeV}$ ) and numerical (via lattice Monte Carlo simulations), to reliably determine these functions. At temperatures below one $\mathrm{GeV}$, relevant for heavy ion collisions, the major qualitative finding has been that there is probably no phase transition at any $T$, only a smooth crossover $[2,3]$. Nevertheless large-scale numerical efforts to determine $p(T)$ and its derivatives for physical QCD in the infinite-volume and continuum limit go on. At temperatures above a few $\mathrm{GeV}$, relevant for cosmology, the system should be addressable with weak-coupling techniques. Perturbation theory does suffer from serious infrared problems, however: besides proceeding in powers of $\alpha_{\mathrm{s}}^{1 / 2}$ rather than $\alpha_{\mathrm{s}}$ [14], it also comes with non-perturbative coefficients starting at the 
order $\alpha_{\mathrm{s}}^{3}$ [15]. Nevertheless it is possible to compile (systematically improvable) phenomenological values for $p(T), p^{\prime}(T)$ and $p^{\prime \prime}(T)$ for temperatures above a few $\mathrm{GeV}$, taking into account the crossing of various quark mass thresholds [16]. By applying similar techniques to the weak sector of the Standard Model, even temperatures up to the TeV range can be reached [17, 18]. Such results do play a role in precision dark matter computations [19], so that studies of the equation of state in the weak-coupling (but slowly convergent) regime indeed do constitute a bridge between hot QCD technologies and cosmology.

\subsection{Transport coefficients}

Transport coefficients are zero-frequency limits of correlators related to components of conserved currents, such as the energy-momentum tensor or approximately conserved particle number currents. Examples of transport coefficients are the shear and bulk viscosities, diffusion coefficients related to various particle species, as well as the electromagnetic conductivity.

In a thermal system, transport coefficients are functions of the temperature just like the pressure and its derivatives. The structure of the weak-coupling series is in principle also similar, except that the leading term is typically proportional to an inverse power of the coupling constants, and already requires a complicated resummation for its correct determination.

Transport coefficients play an important role in the hydrodynamic analysis of heavy ion collision experiments. Indeed viscosities, which parametrize gradient corrections to the ideal form of the energy-momentum tensor, are among the prime quantities that current heavy ion collision experiments attempt to extract from the bulk hydrodynamical behaviour of the expanding fireball. The diffusion coefficient characterizing the movement and kinetic equilibration of heavy quarks in this background represents a theoretically and experimentally clean "hard probe" of the properties of the system. Electromagnetic conductivity determines the fate of the electromagnetic fields that may be generated in non-central collisions. Because of this the theoretical techniques for systematically computing transport coefficients in weakly coupled gauge theories have first been developed in the context of hot QCD some time ago, see e.g. refs. [20,21] and references therein.

Transport coefficients play a role in cosmology as well. For instance, the evolution of density perturbations leading to large-scale structure formation may be sensitive to viscosities, see, e.g., refs. [22, 23]. Remarkably, the bulk viscosity also plays a role even for certain zero-momentum (non-gradient) perturbations [24]. Indeed, weakly coupled scalar fields play an important role in inflationary cosmology, and it is assumed that a significant amount of energy may be stored in the scalar fields. Later on, as the universe enters the radiation dominated epoch, the scalar fields should "decay", i.e. transport their energy to the known particles (electrons, photons, etc); this is called a reheating period. It is possible to imagine scalar fields, however, which are coupled so weakly that they decay later than the inflaton field, or not at all. If the decay is too slow and the scalar field does not get rid of its energy density, it eventually becomes a dominant component, in conflict with observation. Such a situation is met particularly in connection with scalar fields called moduli, and the problem of their slow decay is the "moduli problem".

Practical estimates of the friction coefficient of scalar fields have been presented in refs. [24, 25], based on earlier determinations of the bulk viscosity in weakly coupled scalar field theory [26] and hot QCD [27], respectively. The conclusion from this exercise is that even though a plasma "facilitates" the dissipation of the energy in the scalar field into that in normal radiation, the decay 
remains so slow that it essentially does not have time to take place within the lifetime of the Universe. Therefore thermal corrections quite probably cannot solve the moduli problem [24]. This puts strong constraints on model building involving moduli fields, similar in spirit e.g. to what apply to models leading to a cosmological domain wall problem.

Conductivity [20, 21] also plays a role in cosmology. Large-scale (intergalactic) magnetic fields are being observed, and one possibility is that they are of cosmological origin. Although producing fields with a long enough correlation length is challenging (since there is no QCD phase transition, one needs to evoke physics around the electroweak scale; then the "natural" distance, given by the horizon radius scaled to present day by universe expansion, is only on a planetary scale), certain mechanisms do exist. An example of a recent simulation, in which dissipation through the conductivity plays an important role, as well as further references, can be found in ref. [28].

Chemical equilibration rate is a transport coefficient related to a nearly (but not exactly) conserved particle number density [29]. For instance, it gives the rate at which weakly interacting massive particles attempt to follow the thermal distribution, before eventually failing to do so (cf. fig. 1(left)); as an equation, this dynamics can be represented as

$$
\dot{n}+3 H n=-\Gamma_{\text {chem }}\left(n-n_{\text {eq }}\right)+\mathscr{O}\left(n-n_{\text {eq }}\right)^{2},
$$

where $n$ is some number density, $n_{\mathrm{eq}}$ is its equilibrium value, $H$ is the Hubble rate, and $\Gamma_{\text {chem }}$ is the chemical equilibration rate. Now, the physics of non-relativistic massive particles is non-trivial, manifesting e.g. the so-called Sommerfeld effect, which signals a breakdown of the perturbative series for the pair creation or pair annihilation rate at temperatures $T \lesssim \alpha^{2} M$, where $\alpha$ is a finestructure constant. Contrary to several of the other examples mentioned, in this case the relevant phenomena were first elucidated in the context of cosmology, where the Sommerfeld effect was understood to play an important role, see e.g. refs. [30]-[33]. Subsequently it has also been applied to the question of how fast the number density of charm quarks produced in heavy ion collision experiments approaches its thermal value [34].

Kinetic equilibration rate of massive particles in hot QCD [35] can also be understood as a transport coefficient $[36,37]$. In cosmology, an analogous kinetic equilibration also plays a role, given that many Dark Matter candidates kinetically decouple in a non-relativistic regime, and their momentum distribution dictates the kind of structures that can form [23]. In this case, it appears that the two lines of research have developed independently of each other, without any clear information exhange between hot QCD and cosmology having taken place.

As a final example let us mention the rate of anomalous chirality violation, a transport coefficient related to the axial current. Indeed it plays a role both in heavy ion collision experiments, with the gauge group SU(3) [38], as well as in cosmology, with the gauge group $\mathrm{SU}(2)$ (see e.g. ref. [39] and references therein). The techniques used in both cases are essentially the same; in this case they were first developed on the cosmology side.

\subsection{Thermal particle production}

We turn to the final example, that of thermal particle production, illustrated in fig. 1(middle) as leading to "non-thermal" dark matter or the "weak washout" scenario of leptogenesis. Note that 
the production is "thermal" because it takes place from a thermalized plasma; but the result is nonthermal because the number density of the particles produced remains below its equilibrium value. (If the number density does reach the equilibrium value, the relevant observable is the chemical equilibration rate rather than the particle production rate.)

In heavy ion collision experiments, thermal particle production is among the most obvious quantities to consider. The basic observation is that electromagnetic processes do not have time to take place within the lifetime of the thermal fireball; therefore photons (whether real or virtual) essentially escape the system, never reaching equilibrium. The processes can be illustrated as

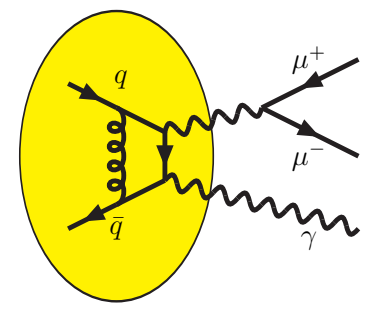

There is a large body of work concerning the computation of the corresponding rates.

What is relevant for the present talk is that the production of such "hard probes" from a QCD plasma is similar to the production of very weakly interacting particles in cosmology. Examples of such particles, which could perhaps contribute to dark matter or leptogenesis, are gravitinos, axions, axinos, and right-handed neutrinos. In the present talk the focus is on the last ones, given that right-handed neutrinos are a well-motivated particle species in any case, since they appear to offer the most natural explanation for the fact that at least two of the left-handed neutrinos have been observed to be massive.

Now, in QCD, the relevant gauge group is SU(3), and the "rungs" of the diagram above are gluons. For right-handed neutrinos, in contrast, the electroweak group $\mathrm{SU}(2) \times \mathrm{U}(1)$ plays a more prominent role, and the rungs of the corresponding diagrams are $W^{ \pm}, Z^{0}$ bosons. However, if we concentrate on temperatures above a few hundred $\mathrm{GeV}$, then the electroweak gauge group is in its "confinement" (or "symmetric") rather than the usual "Higgs" phase, and the difference is inessential from the qualitative point of view. Thereby we again find a platform for a useful exchange of theoretical methods between hot QCD and cosmology.

To be concrete, consider one generation of right-handed neutrinos, with a Majorana spinor field denoted by $\tilde{N}$, a mass parameter denoted by $M$, and a Yukawa coupling denoted by $h_{v}$. Then the most general gauge-invariant and renormalizable addition to the Standard Model Lagrangian has the form

$$
\delta \mathscr{L}=\frac{1}{2} \overline{\tilde{N}}[i \not \partial-M] \tilde{N}-\left[h_{v} \bar{\ell} a_{R} \tilde{\phi} \tilde{N}+\text { H.c. }\right]
$$

Here $\tilde{\phi}=i \sigma_{2} \phi^{*}$ is the Higgs doublet, $\ell$ is a lepton doublet, and $a_{\mathrm{R}}$ is a chiral projector.

Because of the Yukawa term, interactions with Standard Model particles modify the selfenergy of the right-handed neutrinos. Defining a gauge-invariant $4 \times 4$ self-energy matrix in Euclidean signature formally as

$$
\Sigma_{E}(K) \equiv \int_{X} e^{i K \cdot X}\left\langle\left(\tilde{\phi}^{\dagger} a_{\mathrm{L}} \ell\right)(X)\left(\bar{\ell} a_{\mathrm{R}} \tilde{\phi}\right)(0)\right\rangle_{T}, \quad K \equiv\left(k_{n}, \mathbf{k}\right),
$$




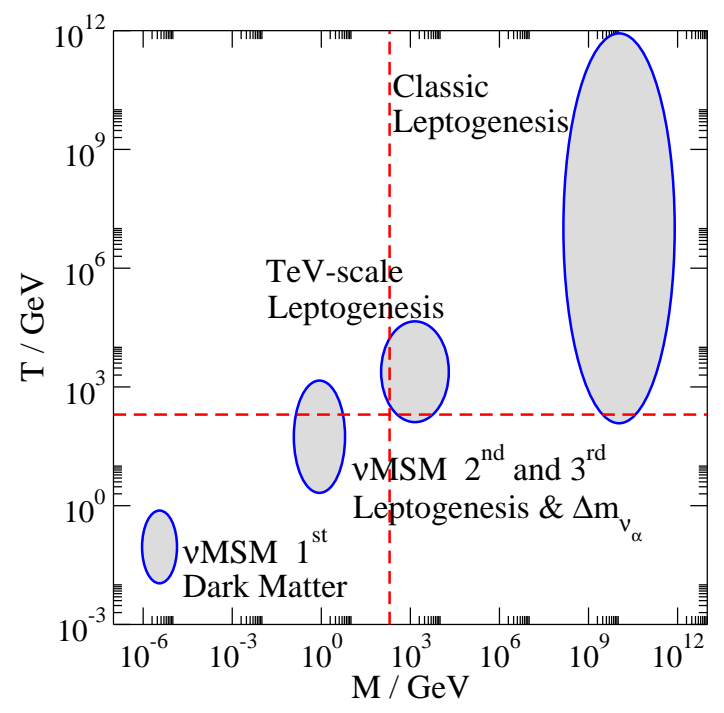

Figure 3: A sketch of some of the parameter regions leading to a cosmologically significant role for righthanded neutrinos. More details on the two islands of the " $v M$ MSM" scenario can be found e.g. in ref. [9], whereas the $\mathrm{TeV}$ scale leptogenesis scenario has been considered e.g. in refs. [41]-[46].

the corresponding spectral function can be obtained from an analytic continuation,

$$
\rho(\mathscr{K}) \equiv \frac{1}{2 i}\left[\Sigma_{E}\left(k_{n} \rightarrow-i\left[k^{0}+i 0^{+}\right], \mathbf{k}\right)-\Sigma_{E}\left(k_{n} \rightarrow-i\left[k^{0}-i 0^{+}\right], \mathbf{k}\right)\right], \quad \mathscr{K} \equiv\left(k^{0}, \mathbf{k}\right) .
$$

A simple exercise (see e.g. ref. [10]) then shows that, as long as the number density of the particles produced is below its equilibrium value, like in fig. 1(middle), their production rate can be written as

$$
\frac{\mathrm{d} N}{\mathrm{~d}^{4} \mathscr{X} \mathrm{d}^{3} \mathbf{k}}=\frac{2 n_{\mathrm{F}}\left(k^{0}\right) \Gamma(\mathscr{K})}{(2 \pi)^{3}}, \quad \Gamma(\mathscr{K})=\frac{\left|h_{v}\right|^{2}}{k^{0}} \operatorname{Tr}\{\mathscr{K} \rho(\mathscr{K})\}+\mathscr{O}\left(\left|h_{v}\right|^{4}\right),
$$

where $n_{\mathrm{F}}$ is the Fermi distribution. Here the four-momentum is on-shell: $\mathscr{K}=\left(\sqrt{k^{2}+M^{2}}, \mathbf{k}\right)$. The process can be illustrated as

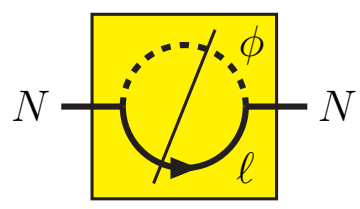

where the diagonal line indicates the cut from eq. (4.4).

Even after taking into account the known left-handed neutrino mass differences and mixing angles, not that much is known about the values of $h_{v}$ and $M$ (the issue is that only the combination $\sim\left|h_{v}\right|^{2} / M$ plays a role at low energies). In fact many different orders of magnitude can be considered; some of the cases are illustrated in fig. 3. For simplicity we focus on the "classic leptogenesis" case here, with $M, \pi T \gg 100 \mathrm{GeV}$; this scenario was originally put forward in ref. [40].

Now, even after restricting to $M, \pi T \gg 100 \mathrm{GeV}$, whereby zero-temperature scales such as $m_{\text {top }}$ or $m_{W^{ \pm}}$play no role, there are still two parameters in the problem, out of which a dimensionless 

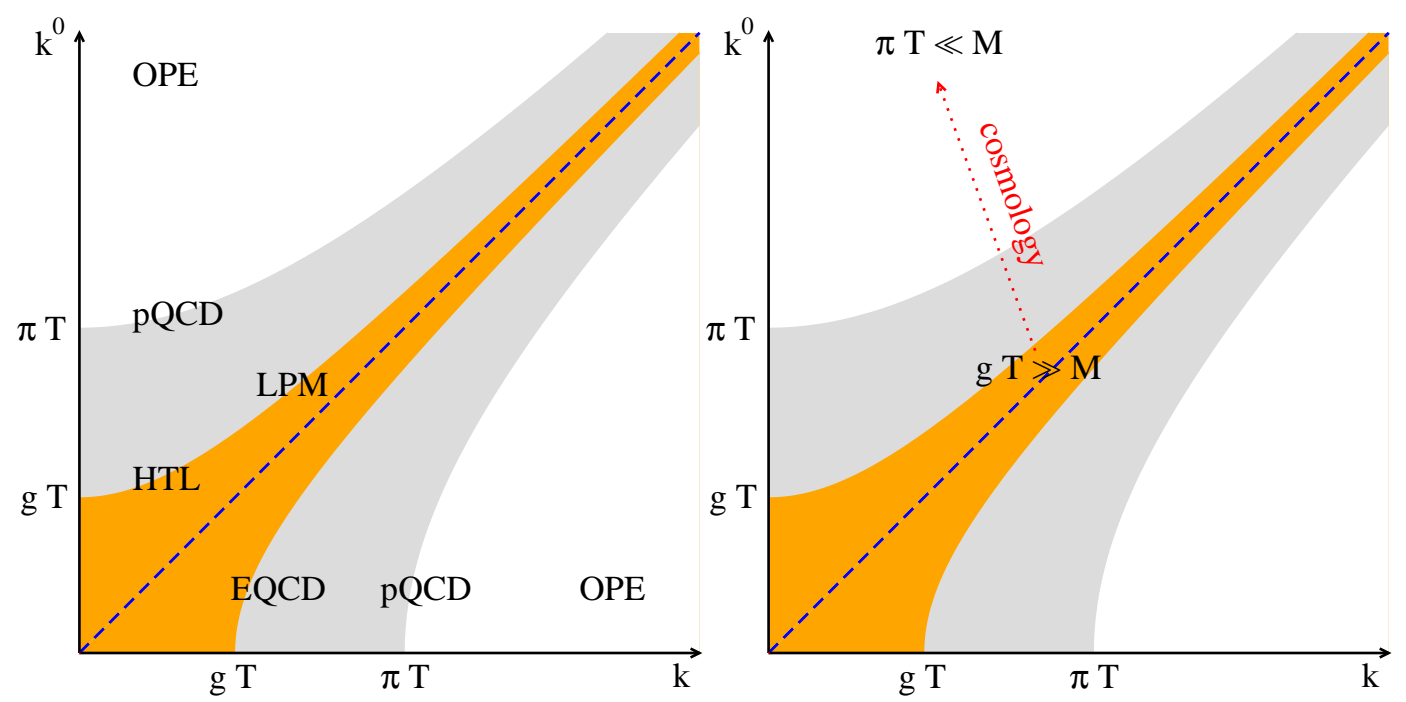

Figure 4: Left: regions of four-momenta $\mathscr{K}=\left(k^{0}, \mathbf{k}\right)$ studied in thermal QCD, with the acronyms representing effective field theories or resummation schemes needed for a systematic treatment. Right: a possible right-handed neutrino trajectory in the early universe. Here $g \equiv \sqrt{4 \pi \alpha}$, where $\alpha$ refers to the strong gauge coupling in QCD and to the weak one in the Standard Model.

ratio $M / \pi T$ can be formed. This implies that there are different regimes, depending on the value of $M / \pi T$ (or, if the full spectrum rather than the total production rate is considered, on $\left(k^{0}, k\right) / \pi T$, where $k \equiv|\mathbf{k}|)$. The different regimes, as have been studied in the case of similar spectral functions in QCD on one hand, and as is relevant for the right-handed neutrino production rate on the other, are illustrated in fig. 4.

The most difficult case turns out to be the initial production in a very early universe, referred to as the "ultrarelativistic" regime: $\pi T \gg M \gg 100 \mathrm{GeV}$. In this case the production rate has been computed systematically to leading order in coupling constants only recently [47, 48, 49]. The techniques used, often referred to as those of the "LPM" regime (for Landau, Pomeranchuk and Migdal) have been inspired by the corresponding hot QCD computation of photon production, presented in refs. $[50,51,52]$. Therefore the recent progress can be viewed as a prime example of a fruitful information exchange between the two fields.

In order to illustrate the practical importance of these developments, we need to introduce two concepts. Traditionally, a "tree-level" computation refers to giving all particles effective ("asymptotic") thermal masses, and then computing the production rate from the lowest-order kinematically allowed diagram. In contrast, a "consistent LO" computation needs to include all processes which contribute at the same order in coupling constants (but not necessarily at the same order in the loop expansion). This requires, in particular, the resummation of the relevant "ladder" diagrams, which do not amount to mass corrections.

A comparison of the results obtained with these two procedures is illustrated in fig. 5. At high temperatures a factor of three is observed between the two results, and at intermediate temperatures the difference is even larger. It remains to be explored how big a practical effect these corrections have in, say, leptogenesis computations, where other ( $\mathrm{CP}$ violating) phenomena also 


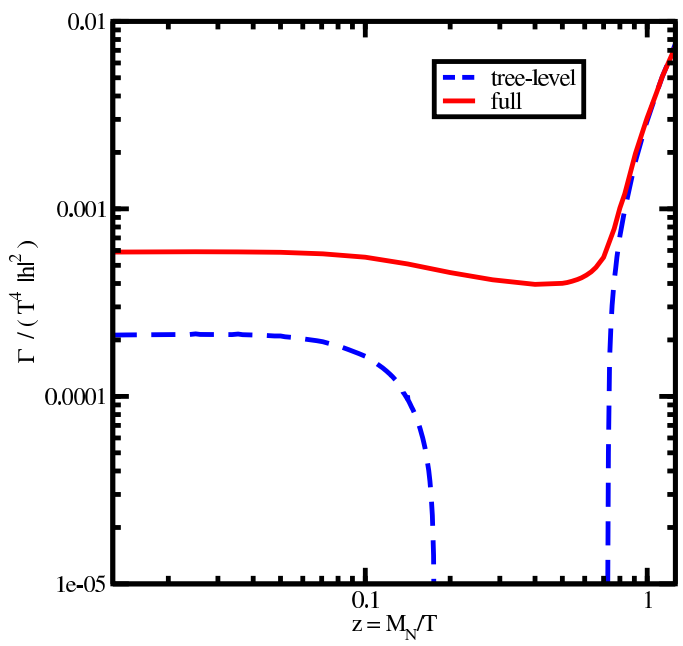

Figure 5: Right-handed neutrino production rate according to ref. [48]. The difference of "tree-level" (thermal effects included through effective masses) and "full" (all loop orders systematically resummed in order to obtain a consistent leading-order result) is substantial at all but the lowest temperatures.

enter the analysis. However, effects of order unity are clearly to be expected.

A simpler case is that of a late universe, referred to as the "non-relativistic" regime: $M \gg$ $\pi T \gg 100 \mathrm{GeV}$. This situation has also been addressed recently [53, 54, 55]; the techniques ("OPE", for the Operator Product Expansion) are again similar to those that had been introduced previously in the context of a hot QCD computation [56].

The difference between the ultrarelativistic and non-relativistic regimes is that whereas in the former case infinitely many loop orders need to be resummed for obtaining a consistent leadingorder result, in the latter case no resummation is needed, and we are already discussing NLO (next-to-leading order) corrections. Moreover, the NLO corrections are argued to be small.

How can we understand this major qualitative difference? When we are considering the nonrelativistic regime then, from the viewpoint of the plasma, $\mathscr{K}^{2}=M^{2}$ is a hard scale. This means that we are in an "ultraviolet" regime, and can expand the result in $\mathscr{O}\left(T^{2} / \mathscr{K}^{2}\right)$ : schematically,

$$
\operatorname{Tr}\{\mathscr{K} \rho(\mathscr{K})\} \sim f_{T=0}^{(2)}\left(\mathscr{K}^{2}\right)+f^{(0)}\left(\mathscr{K}^{2} ; \bar{\mu}^{2}\right)\left\langle\phi^{\dagger} \phi\right\rangle_{T}+\mathscr{O}\left(\frac{T^{4}}{\mathscr{K}^{2}}\right) .
$$

Here the $f$ 's denote Wilson coefficients, which are independent of the temperature but can depend on a factorization scale, $\bar{\mu}$; and thermal corrections only appear through expectation values of gauge-invariant condensates, the lowest-dimensional of which is $\left\langle\phi^{\dagger} \phi\right\rangle_{T}$.

Now, the form of eq. (4.6) implies that even if there are problems with thermal perturbation theory, they are isolated into the computations of static 1-point functions, much as the observables considered in sec. 4.1. This already renders them much simpler than the computations in the ultrarelativistic regime, where genuine 2-point functions need to be addressed; for instance, perturbative resummation techniques removing divergences are available up to the 4-loop level, and the infrared sector could also be handled non-perturbatively with relatively simple lattice Monte Carlo 
simulations if desired [39]. Moreover, irrespective of these issues, dimensional reasons guarantee that thermal corrections are small at low temperatures, suppressed by $\sim T^{2} / M^{2}$ with respect to the leading term in eq. (4.6), so that theoretical errors can be brought under control.

As a concrete example, the complete self-energy in this regime can be expressed as [55]

$$
\begin{aligned}
\mathscr{Z}_{v} \Sigma_{E}(K)= & a_{\mathrm{L}} i K a_{\mathrm{R}}\left\{\frac{1}{(4 \pi)^{2}}\left(\frac{1}{\varepsilon}+\ln \frac{\bar{\mu}^{2}}{K^{2}}+2\right)\right. \\
& +\frac{\left|h_{t}\right|^{2} N_{\mathrm{c}}}{(4 \pi)^{4}}\left(\frac{1}{2 \varepsilon^{2}}-\frac{3}{4 \varepsilon}-\frac{1}{2} \ln ^{2} \frac{\bar{\mu}^{2}}{K^{2}}-\frac{7}{2} \ln \frac{\bar{\mu}^{2}}{K^{2}}-\frac{57}{8}\right) \\
& +\frac{g_{1}^{2}+3 g_{2}^{2}}{(4 \pi)^{4}}\left(-\frac{3}{8 \varepsilon^{2}}+\frac{17}{16 \varepsilon}+\frac{3}{8} \ln ^{2} \frac{\bar{\mu}^{2}}{K^{2}}+\frac{29}{8} \ln \frac{\bar{\mu}^{2}}{K^{2}}+\frac{275}{32}-3 \zeta(3)\right) \\
& \left.+\left[1+\frac{6 \lambda}{(4 \pi)^{2}}\left(\ln \frac{\bar{\mu}^{2}}{K^{2}}+1\right)\right] \frac{\mathscr{Z}_{m}\left\langle\phi^{\dagger} \phi\right\rangle_{T}}{K^{2}}+\mathscr{O}\left(g^{4}, \frac{T^{4}}{K^{4}}\right)\right\}, \\
\mathscr{Z}_{m}\left\langle\phi^{\dagger} \phi\right\rangle_{T}= & \frac{T^{2}}{6}-\frac{T^{2}}{2 \pi} \sqrt{\frac{m_{H}^{2}}{T^{2}}-\frac{g_{1}^{2} m_{\mathrm{D} 1}+3 g_{2}^{2} m_{\mathrm{D} 2}}{16 \pi T}} \\
& +\frac{T^{2}}{48 \pi^{2}}\left\{-6 \lambda\left[\ln \left(\frac{\bar{\mu} e^{\gamma_{\mathrm{E}}}}{4 \pi T}\right)-3\right]-\left|h_{t}\right|^{2} N_{\mathrm{c}} \ln \left(\frac{\bar{\mu} e^{\gamma_{\mathrm{E}}}}{8 \pi T}\right)\right. \\
& \left.+\frac{3\left(g_{1}^{2}+3 g_{2}^{2}\right)}{4}\left[\ln \left(\frac{\bar{\mu} e^{\gamma_{\mathrm{E}}}}{4 \pi T}\right)-\frac{2}{3}-2 \gamma_{\mathrm{E}}-2 \frac{\zeta^{\prime}(-1)}{\zeta(-1)}+4 \ln \left(\frac{2 \pi T}{m_{\mathrm{H}}}\right)\right]\right\}+\ldots,
\end{aligned}
$$

where $\mathscr{Z}_{v}$ and $\mathscr{Z}_{m}$ are renormalization factors; $h_{t}^{2}$ is the top Yukawa coupling; $\lambda$ the Higgs selfcoupling; and $g_{1}^{2}, g_{2}^{2}$ the $\mathrm{U}(1)$ and $\mathrm{SU}(2)$ gauge couplings, respectively. The parameters $m_{\mathrm{D} 1}, m_{\mathrm{D} 2}$, $m_{\mathrm{H}}$ refer to the $\mathrm{U}(1)$ and $\mathrm{SU}(2)$ Debye mass parameters and the thermal Higgs mass parameter, respectively, all of which scale as $\sim g T$ at high temperatures. The corresponding spectral function is obtained from eq. (4.4), and gets a contribution only from terms containing logarithms of $K^{2}$.

In eq. (4.8) the condensate $\mathscr{Z}_{m}\left\langle\phi^{\dagger} \phi\right\rangle_{T}$ has been given up to NNLO, with corrections of relative orders $\mathscr{O}(g)$ and $\mathscr{O}\left(g^{2}\right)$, or $\mathscr{O}\left(\alpha^{1 / 2}\right)$ and $\mathscr{O}(\alpha)$ in the language of sec. 4.1. These corrections are of peculiar types, containing functions that are non-analytic in the coupling constants. Inserting numerical values, it also appears that the practical convergence of the series is marginal at best. Nevertheless, as mentioned, the thermal correction as a whole is power-suppressed with respect to the well-convergent vacuum contribution. So, in this regime, techniques formulated originally for hot QCD [56] allow us to obtain reliable results also for cosmology [53, 54, 55].

\section{Conclusions}

The purpose of this talk has been to illustrate that theoretical work in hot QCD, originally motivated by heavy ion collision experiments, may find unexpected applications in cosmology, just by changing the gauge group from $\mathrm{SU}(3)$ into $\mathrm{SU}(2) \times \mathrm{U}(1)$, and vice versa. It is at least in this wider sense that hot QCD and cosmology should continue to inspire each other for years to come. There are also specific cases in which a more direct link could exist (cf. sec. 2), however in these cases it remains to be seen whether nature has made use of these possibilities. 


\section{Acknowledgements}

This work was partly supported by the Swiss National Science Foundation (SNF) under grant 200021-140234.

\section{References}

[1] E. Witten, Cosmic Separation of Phases, Phys. Rev. D 30 (1984) 272.

[2] P. de Forcrand and O. Philipsen, The chiral critical line of $N_{\mathrm{f}}=2+1$ QCD at zero and non-zero baryon density, JHEP 01 (2007) 077 [hep-lat/0607017].

[3] Y. Aoki, G. Endrodi, Z. Fodor, S.D. Katz and K.K. Szabo, The order of the Quantum Chromodynamics transition predicted by the Standard Model of particle physics, Nature 443 (2006) 675 [hep-lat/0611014].

[4] D.J. Schwarz, Evolution of gravitational waves through cosmological transitions, Mod. Phys. Lett. A 13 (1998) 2771 [gr-qc/9709027].

[5] N. Seto and J. Yokoyama, Probing the equation of state of the early universe with a space laser interferometer, J. Phys. Soc. Jap. 72 (2003) 3082 [gr-qc/0305096].

[6] L.A. Boyle and P.J. Steinhardt, Probing the early universe with inflationary gravitational waves, Phys. Rev. D 77 (2008) 063504 [astro-ph/0512014].

[7] M. Hindmarsh and O. Philipsen, WIMP dark matter and the QCD equation of state, Phys. Rev. D 71, 087302 (2005) [hep-ph/0501232].

[8] M. Laine and M. Shaposhnikov, Sterile neutrino dark matter as a consequence of VMSM-induced lepton asymmetry, JCAP 06 (2008) 031 [0804.4543].

[9] L. Canetti, M. Drewes, T. Frossard and M. Shaposhnikov, Dark Matter, Baryogenesis and Neutrino Oscillations from Right Handed Neutrinos, 1208.4607.

[10] T. Asaka, M. Laine and M. Shaposhnikov, On the hadronic contribution to sterile neutrino production, JHEP 06 (2006) 053 [hep-ph/0605209].

[11] B.B. Brandt, A. Francis, H.B. Meyer and H. Wittig, Thermal Correlators in the $\rho$ channel of two-flavor QCD, 1212.4200 .

[12] P. Naselsky, C.H. Christensen, P.R. Christensen, P.H. Damgaard, A. Frejsel, J.J. Gaardhøje, A. Hansen, M. Hansen, J. Kim, O. Verkhodanov, U.A. Wiedemann, Morphology of High-Multiplicity Events in Heavy Ion Collisions, Phys. Rev. C 86 (2012) 024916 [1204.0387].

[13] P. Gondolo and G. Gelmini, Cosmic abundances of stable particles: Improved analysis, Nucl. Phys. B 360 (1991) 145.

[14] J.I. Kapusta, Quantum Chromodynamics at high temperature, Nucl. Phys. B 148 (1979) 461.

[15] A.D. Linde, Infrared problem in thermodynamics of the Yang-Mills gas, Phys. Lett. B 96 (1980) 289.

[16] M. Laine and Y. Schröder, Quark mass thresholds in QCD thermodynamics, Phys. Rev. D 73 (2006) 085009 [hep-ph/0603048].

[17] A. Gynther and M. Vepsäläinen, Pressure of the standard model at high temperatures, JHEP 01 (2006) 060 [hep-ph/0510375]. 
[18] A. Gynther and M. Vepsäläinen, Pressure of the standard model near the electroweak phase transition, JHEP 03 (2006) 011 [hep-ph/0512177].

[19] G. Steigman, B. Dasgupta and J.F. Beacom, Precise Relic WIMP Abundance and its Impact on Searches for Dark Matter Annihilation, Phys. Rev. D 86 (2012) 023506 [1204.3622].

[20] P.B. Arnold, G.D. Moore and L.G. Yaffe, Transport coefficients in high temperature gauge theories. 1. Leading log results, JHEP 11 (2000) 001 [hep-ph/0010177].

[21] P.B. Arnold, G.D. Moore and L.G. Yaffe, Transport coefficients in high temperature gauge theories. 2. Beyond leading log, JHEP 05 (2003) 051 [hep-ph/0302165].

[22] Steven Weinberg, Gravitation and Cosmology (Wiley, New York, 1972).

[23] S. Hofmann, D.J. Schwarz and H. Stöcker, Damping scales of neutralino cold dark matter, Phys. Rev. D 64 (2001) 083507 [astro-ph/0104173].

[24] D. Bödeker, Moduli decay in the hot early universe, JCAP 06 (2006) 027 [hep-ph/0605030].

[25] M. Laine, On bulk viscosity and moduli decay, Prog. Theor. Phys. Suppl. 186 (2010) 404 [1007.2590].

[26] S. Jeon and L.G. Yaffe, From quantum field theory to hydrodynamics: Transport coefficients and effective kinetic theory, Phys. Rev. D 53 (1996) 5799 [hep-ph/9512263].

[27] P.B. Arnold, C. Dogan and G.D. Moore, The bulk viscosity of high-temperature QCD, Phys. Rev. D 74 (2006) 085021 [hep-ph/0608012].

[28] M. Dvornikov and V. B. Semikoz, Lepton asymmetry growth in symmetric phase of electroweak plasma with hypermagnetic fields versus its washing out by sphalerons, 1212.1416.

[29] D. Bödeker and M. Laine, Heavy quark chemical equilibration rate as a transport coefficient, JHEP 07 (2012) 130 [1205.4987].

[30] J. Hisano, S. Matsumoto, M. Nagai, O. Saito and M. Senami, Non-perturbative effect on thermal relic abundance of dark matter, Phys. Lett. B 646 (2007) 34 [hep-ph/0610249].

[31] J.L. Feng, M. Kaplinghat and H.-B. Yu, Sommerfeld Enhancements for Thermal Relic Dark Matter, Phys. Rev. D 82 (2010) 083525 [1005.4678].

[32] A. Hryczuk, R. Iengo and P. Ullio, Relic densities including Sommerfeld enhancements in the MSSM, JHEP 03 (2011) 069 [1010.2172].

[33] A. Strumia, Sommerfeld corrections to type-II and III leptogenesis, Nucl. Phys. B 809 (2009) 308 [0806.1630].

[34] D. Bödeker and M. Laine, Sommerfeld effect in heavy quark chemical equilibration, JHEP 01 (2013) 037 [1210.6153].

[35] G.D. Moore and D. Teaney, How much do heavy quarks thermalize in a heavy ion collision?, Phys. Rev. C 71 (2005) 064904 [hep-ph/0412346].

[36] J. Casalderrey-Solana and D. Teaney, Heavy quark diffusion in strongly coupled $\mathscr{N}=4$ Yang Mills, Phys. Rev. D 74 (2006) 085012 [hep-ph/0605199].

[37] S. Caron-Huot, M. Laine and G.D. Moore, A way to estimate the heavy quark thermalization rate from the lattice, JHEP 04 (2009) 053 [0901.1195].

[38] G.D. Moore and M. Tassler, The Sphaleron Rate in SU(N) Gauge Theory, JHEP 02 (2011) 105 [1011.1167]. 
[39] M. D’Onofrio, K. Rummukainen and A. Tranberg, The Sphaleron Rate through the Electroweak Cross-over, JHEP 08 (2012) 123 [1207.0685].

[40] M. Fukugita and T. Yanagida, Baryogenesis Without Grand Unification, Phys. Lett. B 174 (1986) 45.

[41] T. Hambye, Leptogenesis at the TeV scale, Nucl. Phys. B 633 (2002) 171 [hep-ph/0111089].

[42] M. Senami and K. Yamamoto, Lepton flavor violation with supersymmetric Higgs triplets in TeV region for neutrino masses and leptogenesis, Phys. Rev. D 69 (2004) 035004 [hep-ph/0305203].

[43] A. Pilaftsis and T.E.J. Underwood, Resonant leptogenesis, Nucl. Phys. B 692 (2004) 303 [hep-ph/0309342].

[44] S. Dar, S. Huber, V.N. Senoguz and Q. Shafi, TeV scale leptogenesis with heavy neutrinos, Phys. Rev. D 69 (2004) 077701 [hep-ph/0311129].

[45] A. Abada, H. Aissaoui and M. Losada, A model for leptogenesis at the TeV scale, Nucl. Phys. B 728 (2005) 55 [hep-ph/0409343].

[46] N. Sahu and U.A. Yajnik, Gauged B-L symmetry and baryogenesis via leptogenesis at TeV scale, Phys. Rev. D 71 (2005) 023507 [hep-ph/0410075].

[47] D. Besak and D. Bödeker, Hard Thermal Loops for Soft or Collinear External Momenta, JHEP 05 (2010) 007 [1002.0022].

[48] A. Anisimov, D. Besak and D. Bödeker, Thermal production of relativistic Majorana neutrinos: Strong enhancement by multiple soft scattering, JCAP 03 (2011) 042 [1012.3784].

[49] D. Besak and D. Bödeker, Thermal production of ultrarelativistic right-handed neutrinos: Complete leading-order results, JCAP 03 (2012) 029 [1202.1288].

[50] P.B. Arnold, G.D. Moore and L.G. Yaffe, Photon emission from ultrarelativistic plasmas, JHEP 11 (2001) 057 [hep-ph/0109064].

[51] P.B. Arnold, G.D. Moore and L.G. Yaffe, Photon emission from quark gluon plasma: Complete leading order results, JHEP 12 (2001) 009 [hep-ph/0111107].

[52] P.B. Arnold, G.D. Moore and L.G. Yaffe, Photon and gluon emission in relativistic plasmas, JHEP 06 (2002) 030 [hep-ph/0204343].

[53] A. Salvio, P. Lodone and A. Strumia, Towards leptogenesis at NLO: the right-handed neutrino interaction rate, JHEP 08 (2011) 116 [1106.2814].

[54] M. Laine and Y. Schröder, Thermal right-handed neutrino production rate in the non-relativistic regime, JHEP 02 (2012) 068 [1112.1205].

[55] M. Laine, Thermal right-handed neutrino self-energy in the non-relativistic regime, 1209.2869.

[56] S. Caron-Huot, Asymptotics of thermal spectral functions, Phys. Rev. D 79 (2009) 125009 [0903.3958]. 